\title{
Development of an HTS Magnet for Ultra-compact MRI System: Optimization using Genetic Algorithm (GA) Method
}

Boyang Shen, Yavuz Öztürk, Wei Wu, Li Lu, Jie Sheng, Zhen Huang, Yujia Zhai, Yupeng Yuan, Weishu Wang, Jun Yin, David Menon, Ari Ercole, Adrian Carpenter, Thomas Painter, Chao Li, James Gawith, Jun Ma, Jiabin Yang, Michael Parizh, and Tim Coombs

Abstract - This paper presents the design of an HTS magnet for an ultra compact MRI system, potentially for the rapid and early diagnosis of brain trauma. Early diagnosis and therapy stratification can reduce the risk for critically brain ill patients with the use of near patient imaging, and can aid with precision medicine. High temperature superconductors (HTS) have the ability to carry large currents in the cryogen free contrition, which can make the MRI system even smaller and lighter. The design and Genetic Algorithm (GA) optimization were based on the FEM package COMSOL Multiphysics with the LiveLink for MATLAB, together with the GA module in the MATLAB optimization toolbox. The relatively thick HTS tape, ST-12-L from the Shanghai Superconductor Technology ${ }^{\circledR}$ was chosen and that made more difficulty on optimization process. Genetic Algorithm method improved the optimization performance, and the uniformity achieved $2.36 \mathrm{ppm}$ in a $10 \times 10 \times 10 \mathrm{~cm}$ DSV. Two cases of the end double-pancake were compared. Further sensitivity studies were performed on the homogeneity with its relationship to the magnet length, and the thickness of HTS tape.

Index Terms - High temperature superconductor (HTS), Magnetic resonance imaging (MRI), HTS magnet, Genetic Algorithm (GA), Finite element method.

\section{INTRODUCTION}

Since the 1990s, the combination of high temperature superconductor (HTS) and magnetic resonance imaging (MRI) has been aroused great interests in both the

This work was supported by the Engineering and Physical Sciences Research Council (EPSRC) under Grant EP/R042918/1 (Corresponding author: Jiabin Yang)

B. Shen, Y Öztürk, C. Li, J. Gawith, J. Ma, J. Yang, and T. Coombs are with the Electrical Engineering Division, Department of Engineering, University of Cambridge, Cambridge CB3 0FA, U.K. (e-mail: bs506@cam.ac.uk; jy391@cam.ac.uk).

W. Wu, L. Lu, J. Sheng, and Z. Huang are with the School of Electronic Information and Electrical Engineering, Shanghai Jiao Tong University, Shanghai 200240, China (e-mail: wei.wu@sjtu.edu.cn).

Y. Zhai is with the College of Electrical and Information Engineering, Hunan University, Changsha, 410082, China (e-mail: yz378@hnu.edu.cn).

Y. Yuan is with the Reliability Engineering Institute, School of Energy and Power Engineering, Wuhan University of Technology, Wuhan 430063, China (e-mail: ypyuan@whut.edu.cn).

W. Wang, and J. Yin are with the School of Electric Power, North China University of Water Resources and Electric Power, Zhengzhou 450045, China (e-mail: wangweishu@ncwu.edu.cn).

D. Menon, and A. Ercole are with the Department of Medicine, Cambridge CB2 0QQ, U.K. (e-mail: dkm13@ @am.ac.uk).

A. Carpenter is with the Department of Clinical Neurosciences, Cambridge CB2 0QQ, U.K. (e-mail: tac12@ cam.ac.uk).

T. Painter is with the National High Magnetic Laboratory, Tallahassee, FL 32310 USA, and also with the Florida State University, Tallahassee, FL 32306 USA (e-mail:painter@magnet.fsu.edu).

M. Parizh is with General Electric - Global Research, One Research Circle, Bldg. MR157 Niskayuna, NY 12309, USA (e-mail: parizh@ge.com). superconductivity and MRI communities. MRI [1-3] and electrical impedance tomography (EIT) [4-8] are the sensitive in vivo imaging technologies with soft-tissue contrast, but due to EIT's ill-posed inverse problem causes lower spatial resolution, MRI is still the only commercial and chemically sensitive imaging of soft tissue lesions with high-resolution.

The price of liquid helium has increased significantly as cryogenic applications such as low temperature superconductor (LTS) applications use more than 50 million cubic meters of gaseous helium every year [9], and the consistent increases of helium prices also affects the MRI market. Therefore, the HTS based MRI scanners become more favorable as they do not require helium cryogen. In the helium cryogen free system, the superconducting magnets made by HTS tapes are able to carry high electrical current density and hence generate properly high magnetic field. The HTS also offers MRI new advantage of reducing the size and weight, which contributes MRI with compact system integration and even the mobility for emergency diagnosis.

The first HTS based MRI magnet was presented in 1999, in the same time with early commercial production of HTS conductors [10]. Early HTS based MRI magnets were manufactured using Bi-2223 tape conductors, which showed excellent electromagnetic performance [11-14]. The Second Generation (2G) Yttrium-Barium-Copper-Oxide (YBCO) HTS tape wound MRI magnet also presented satisfactory magnetic profile for commercial MRIs [15]. Comprehensive reviews of the development of HTS MRI can be found in $[16,17]$.

This paper presents the design of an HTS magnet for an ultra-compact MRI system (example shown in Fig. 1), which is primarily for brain scan. In order to obtain high quality imaging

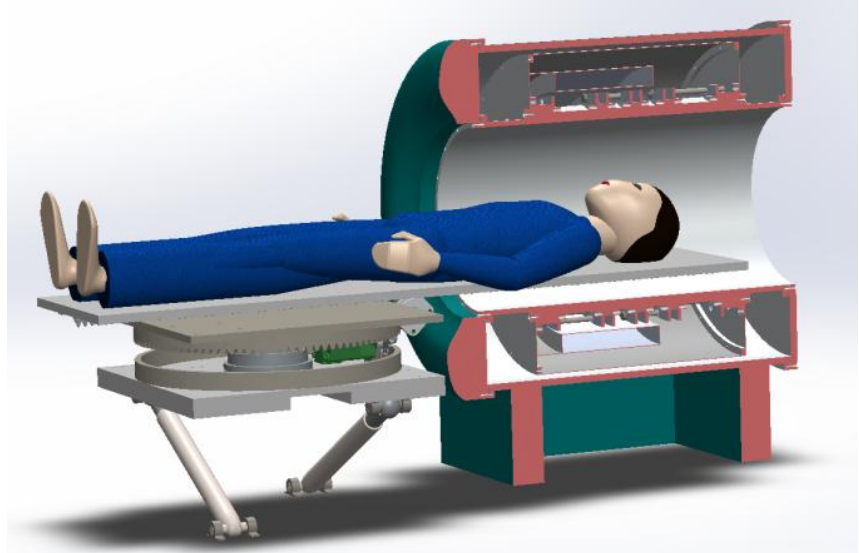

Fig. 1. An ultra-compact MRI system is potentially for the rapid and early diagnosis of brain trauma, where an HTS magnet is a key component to achieve high quality imaging. 


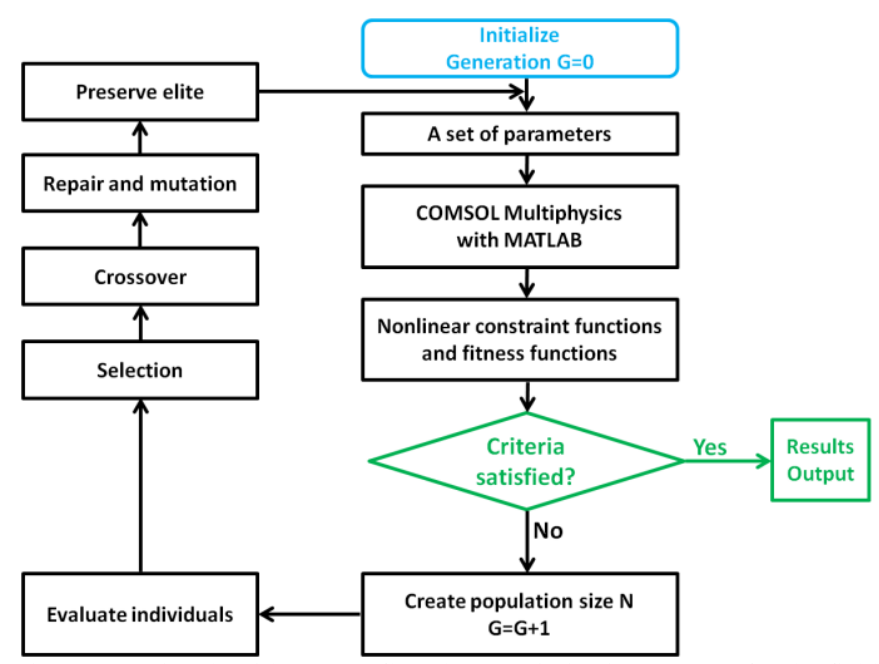

Fig. 2. Genetic Algorithm (GA) flowchart: optimization strategy for the field uniformity of HTS magnet.

TABLE I. PARAMETERS FOR THE HTS MAGNET

\begin{tabular}{ll}
\hline \hline Parameters & Value \\
\hline Tape width & $12 \mathrm{~mm}$ \\
Tape total thickness & $230 \mu \mathrm{m}$ \\
Total tape length & $2.85 \mathrm{~km}$ \\
Number of turns (each double-pancake coil & $75,86,125$ turns \\
from center to end) & \\
HTS Coil inner diameter & $58 \mathrm{~cm}$ \\
Magnet length (distance between end coils' & $54.5 \mathrm{~cm}$ \\
inner edge) & \\
Operating temperature & $10 \mathrm{~K}$ \\
Operating current (ideal) & $1000 \mathrm{~A}$ \\
Central field & $1.6 \mathrm{~T}$ \\
Stored energy & $239 \mathrm{~kJ}$ \\
\hline \hline
\end{tabular}

of human skull, both the strength and uniformity are highly required. The $2 \mathrm{G}$ Rare-earth barium-copper-oxide (ReBCO) HTS tape (ST-12-L), from the Shanghai Superconductor Technology ${ }^{\circledR}$ was selected to implement the superconducting magnet. As the high aspect-ratio dimension of 2G HTS tape, the optimization to achieve excellent homogeneity of magnetic field is very challenging, especially with the thicker HTS tapes (e.g. the total thickness of ST-12-L is approximately $230 \mu \mathrm{m}$ ). Here we introduce a new approach for HTS magnet design using the Genetic Algorithm method implemented into the platform of the Finite Element Method (FEM) package COMSOL Multiphysics with the LiveLink for MATLAB. The optimization performance was improved by Genetic Algorithm method, where the uniformity was able to reach $2.36 \mathrm{ppm}$ in a $10 \times 10 \times 10 \mathrm{~cm}$ diameter of spherical volume (DSV). The sensitivity studies on uniformity with different parameters were carried out, such as the new sensitivity study on the thickness of HTS tape. The approach, results, and analysis of this paper could be potentially helpful for the future design of the HTS magnet for MRI scanners.

\section{MethodOLOGY}

\section{A. Genetic Algorithm (GA)}

Genetic Algorithm (GA) is one of the stochastic optimization methods using global direct search, which is inspired by the natural evolution theory raised by Darwin [18]. GA differs from other stochastic methods such as Simulated Annealing (SA) because GA uses the concept of population, where multiple candidates are simultaneously considered instead of calculating a single solution through each iteration

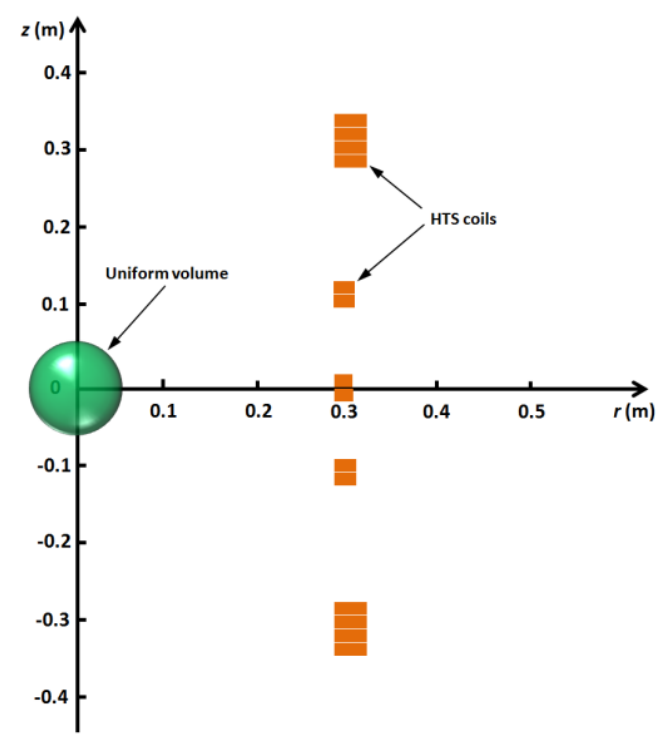

Fig. 3. The basic configuration of the HTS magnet: 5-set of double-pancake HTS coils.

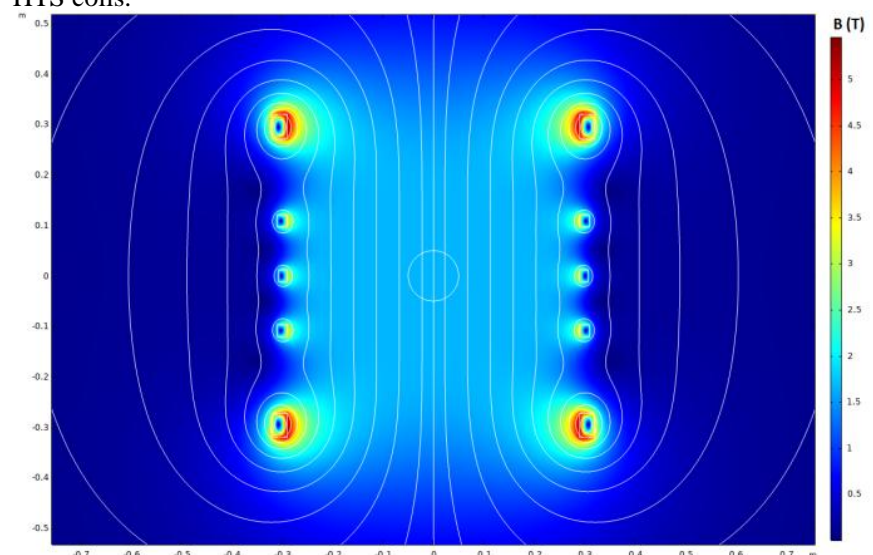

Fig. 4. Overall magnetic profile from the HTS magnet (2-layer end doublepancake case), with the ideal case of transport current $1000 \mathrm{~A}$.

$[18,19]$. GA performs the natural selection to pick the fittest individuals, which survive and evolve towards a better generation. Genetic Algorithm has the capability to solve large-scale and multi-parameter optimization problems at a reasonably fast speed [20]. The concept of GA has been applied to optimize the magnetic field of the LTS magnets for MRI scanners [21, 22], but for HTS magnets using GA optimization literatures have not presented in detail yet.

Our optimization strategy for the field uniformity of HTS magnet is shown in Fig. 2. The advantage of FEM package COMSOL Multiphysics with the LiveLink for MATLAB was that all the settings, the process of FEM solving, input data and output data can be flexibly controlled by MATLAB scripts. The optimization iterations were realized using the FEM MATLAB scripts together with the MATLAB optimization toolbox for Genetic Algorithm. First, GA solver initialized to run with a default set of parameters, and obtained the results of physical properties, such as the magnetic profile from the magnet. After that the results were tested by the nonlinear constraint functions and fitness functions. If the criteria achieved, the optimization iterations stopped; otherwise, the iterations continued to for the purpose of improving the results to reach the targets through repetitive selection, crossover, mutation, and elite preservation, until targets achieved. 


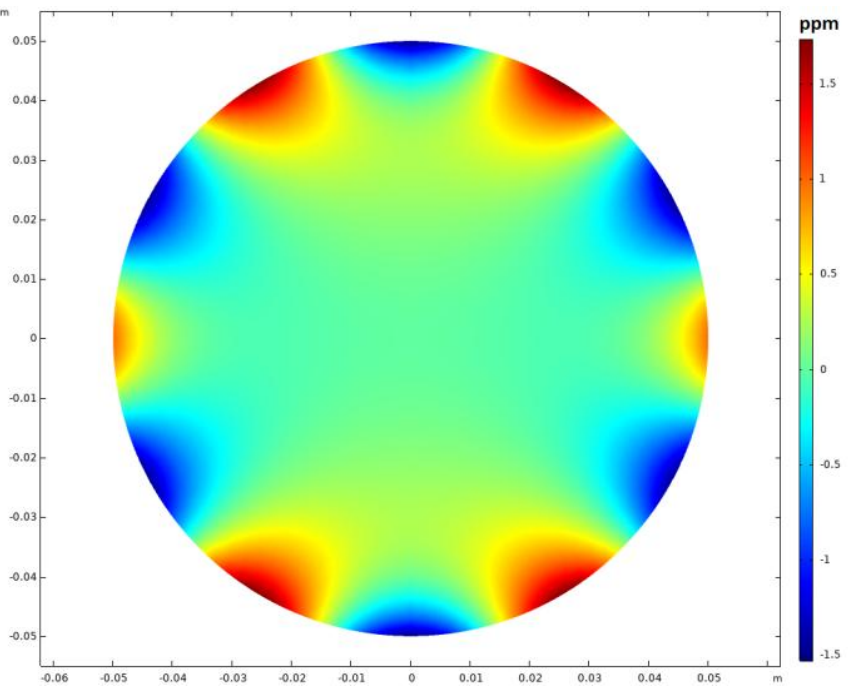

Fig. 5. The magnetic field inhomogeneity in a $10 \mathrm{~cm}$ DSV (1-layer end double-pancake case).

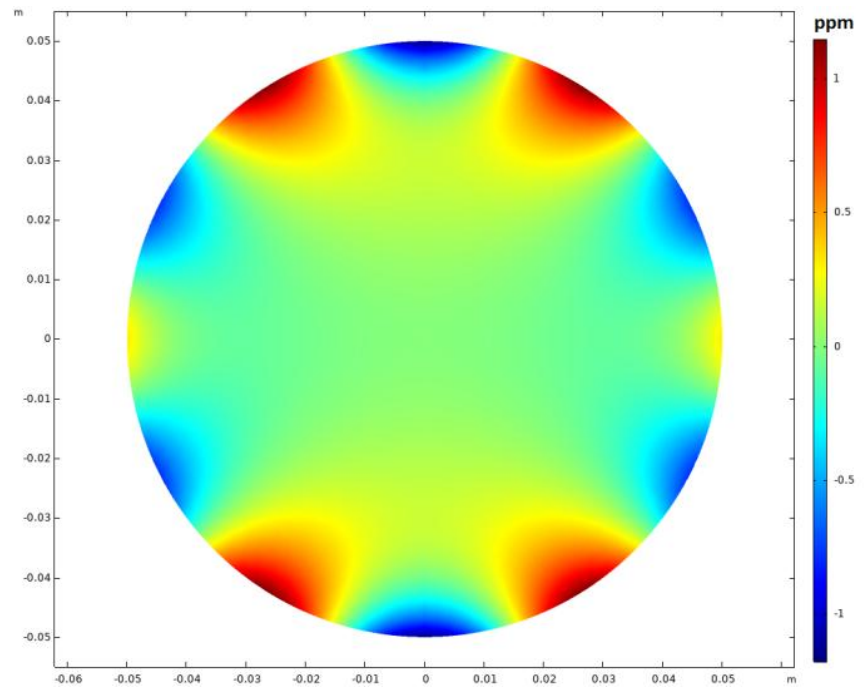

Fig. 6. The magnetic field inhomogeneity in a $10 \mathrm{~cm}$ DSV (2-layer end double-pancake case).

\section{B. HTS Magnet Modeling and optimization}

The basic configuration of the HTS magnet was 5-set of double-pancake HTS coils, and their locations are shown in Fig. 3. The target was the inhomogeneity (ppm, peak-to-peak) as small as possible in a $10 \times 10 \times 10 \mathrm{~cm}$ DSV. The HTS tape ST-12-L (12 mm wide, $230 \mu \mathrm{m}$ thick, minimum critical current $I_{c} 480 \mathrm{~A}$ at $77 \mathrm{~K}$ ) from the Shanghai Superconductor Technology ${ }^{\circledR}$ was chosen as the single element for optimization. The advantages of this relatively thick tape are the decent current carrying capability, the strong mechanical performance and the relatively less sensitive to the anisotropic field dependence, which are favorable for the design of high field magnets, however, at the cost of extra difficulty in the optimization of field uniformity.

The HTS magnet was operated by the driven mode. The inner diameters of all the HTS coils are fixed at $58 \mathrm{~cm}$. The end coils can be either 1-layer double-pancake, or 2-layer double-pancake (series-connected two double-pancake coils). Other coils were fixed as 1-layer double-pancake structure. Each HTS coil's location at z-direction, as well as each HTS coil's number of turns were set as variables. As the real HTS

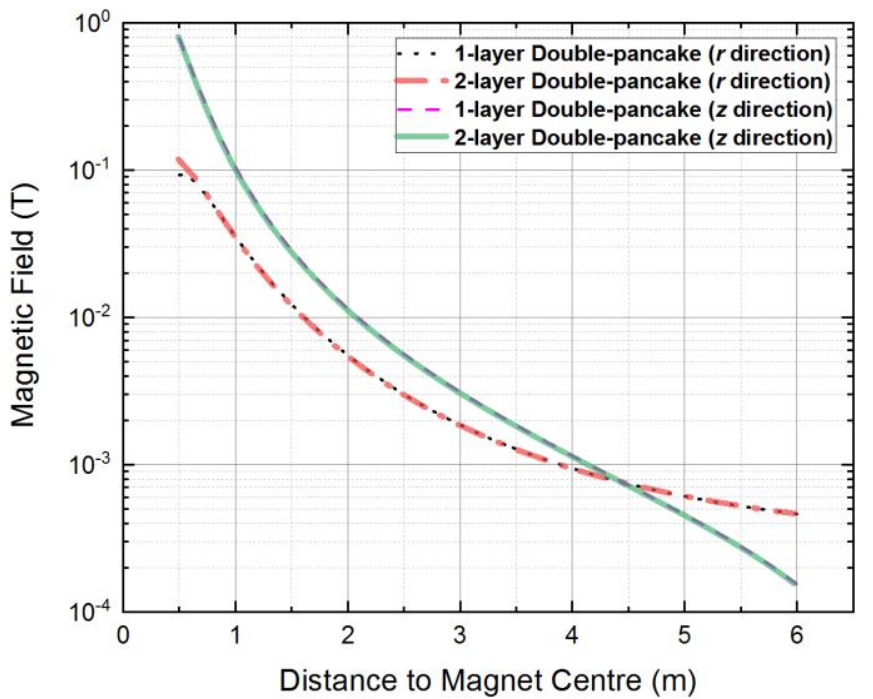

Fig. 7. Stray field (unshielded) in the $r$-direction and $z$-direction, with the ideal case of transport current $1000 \mathrm{~A}$.

magnet will eventually implemented, the minimum step of geometry optimization was set as $0.1 \mathrm{~mm}$, which could be realistic for manufacture.

In the MATLAB GA Optimization tool box, we set the population size 50, the generation 100 , crossover fraction 0.8 . The constraint functions were used primarily to meet the requirement of magnetic field strength, and the fitness functions are used to meet the field uniformity (to achieve the minimum inhomogeneity). Anisotropic field dependence and temperature dependence were not considered at the first stage, as the strength and uniformity of magnetic field are the first to test the feasibility of this HTS magnet with a specific thick HTS tape.

\section{RESULTS AND DISCUSSION}

After maximum iterations of optimization, as well as refining the boundary of parameters for several stages, the GA optimization output the best results. Fig. 4 shows an example of the overall magnetic profile from the HTS magnet (2-layer end double-pancake case), with the ideal case of transport current $1000 \mathrm{~A}$. It can be seen that the magnetic field was highly uniform in the centre part. To be more precise, Fig. 5 presents the magnetic field inhomogeneity (1-layer end double-pancake case) in a $10 \mathrm{~cm}$ DSV was $3.30 \mathrm{ppm}$, while Fig. 6 presents the inhomogeneity (2-layer end doublepancake case) in a $10 \mathrm{~cm}$ DSV was $2.36 \mathrm{ppm}$. By comparison of Fig. 5 and Fig. 6, the inhomogeneity harmonics in the $r$-direction with 2-layer end double-pancake case were relatively smaller than those with 1-layer end double-pancake case.

Fig. 7 provides the information on the stray field (unshielded) in the $r$-direction and $z$-direction, for both the 1-layer and 2-layer end double-pancake cases, with the ideal case of transport current at $1000 \mathrm{~A}$. It can be seen that for both the $r$-direction and $z$-direction, the difference between 1-layer and 2-layer end double-pancake cases was not significant. The 30 Gauss line was approximately at $2.5 \mathrm{~m}$ in $r$-direction, and $3 \mathrm{~m}$ in $z$-direction. The 5 Gauss line was approximately at 5.6 $\mathrm{m}$ in $r$-direction, and $4.8 \mathrm{~m}$ in $z$-direction. 


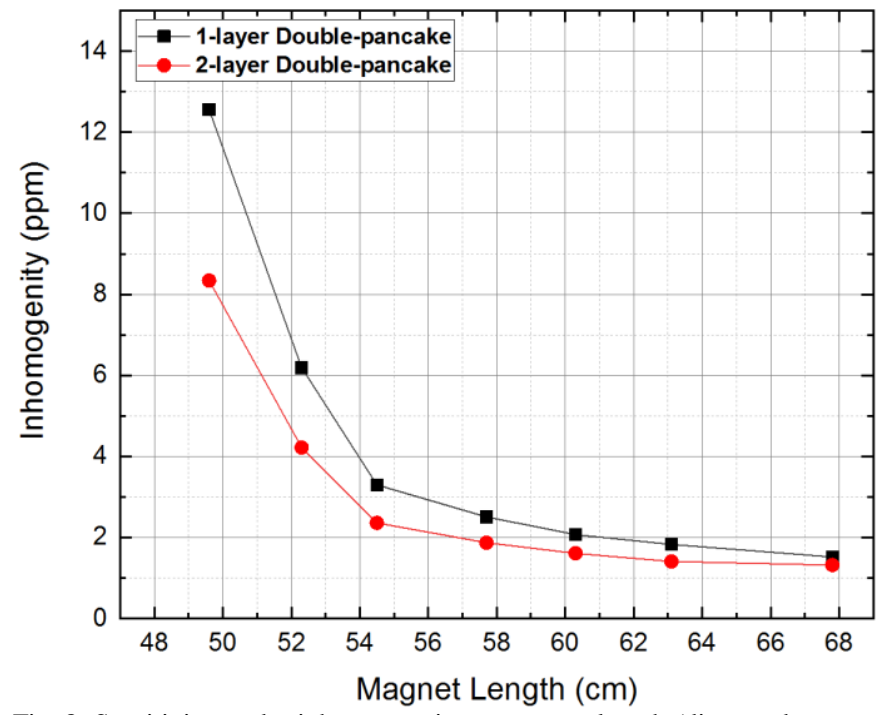

Fig. 8. Sensitivity study: inhomogeneity vs magnet length (distance between end coils' inner edge).

\section{SENSITIVITY STUDIES}

In this section, the sensitivity studies were carried out, for the relationship between the homogeneity in a $10 \mathrm{~cm}$ DSV and (1) magnet length, and (2) thickness of HTS tape. These sensitivity studies were on the basis of the same optimization strategy described in the above sections.

Fig. 8 shows the relationship between the homogeneity and magnet length (distance between end coils' inner edge), for both the cases of 1-layer and 2-layer end double-pancake. It is obvious that for various magnet lengths, the inhomogeneity of the 2-layer end double-pancake case was always smaller than 1-layer end double-pancake case. For example, the with the magnet length $49.6 \mathrm{~cm}$, the inhomogeneity of the 2-layer end double-pancake case was 8.34 ppm while the inhomogeneity of the 1-layer end double-pancake case was $12.56 \mathrm{ppm}$. The difference between two cases became smaller with the increasing magnet length, e. g. at magnet length $67.8 \mathrm{~cm}$ the the inhomogeneity of the 2-layer end double-pancake case was $1.32 \mathrm{ppm}$ and the inhomogeneity of the 1-layer end doublepancake case was $1.52 \mathrm{ppm}$, whose difference was only $0.2 \mathrm{ppm}$. It can be seen for both cases that the decreasing trends of inhomogeneity were very sharp before magnet length $54.5 \mathrm{~cm}$, and afterwards the homogeneity improvement was less obvious.

Fig. 9 presents the relationship between the homogeneity and the thickness of HTS tape, for both the 1-layer and 2-layer end double-pancake cases. The magnet length was fixed at $54.5 \mathrm{~cm}$. For both cases, the thickness of HTS tape $50 \mu \mathrm{m}, 100$ $\mu \mathrm{m}, 230 \mu \mathrm{m}, 300 \mu \mathrm{m}, 400 \mu \mathrm{m}$ were tested to reach their optimum homogeneity. If using the thinnest $50 \mu \mathrm{m}$ tape, both the 1-layer and 2-layer end double-pancake cases were able to achieve the inhomogeneity less than $1 \mathrm{ppm}$. However, with the increment of tape thickness, the difficulty to realize good homogeneity increased significantly. This phenomenon appeared in both case, and that of 1-layer end double-pancake case was more noticeable than that of 2-layer end doublepancake case. As shown in Fig. 9, for both case the inhomogeneities were both over $10 \mathrm{ppm}$ if using the $400 \mu \mathrm{m}$ HTS tape. A thicker HTS tape offers a better current carrying

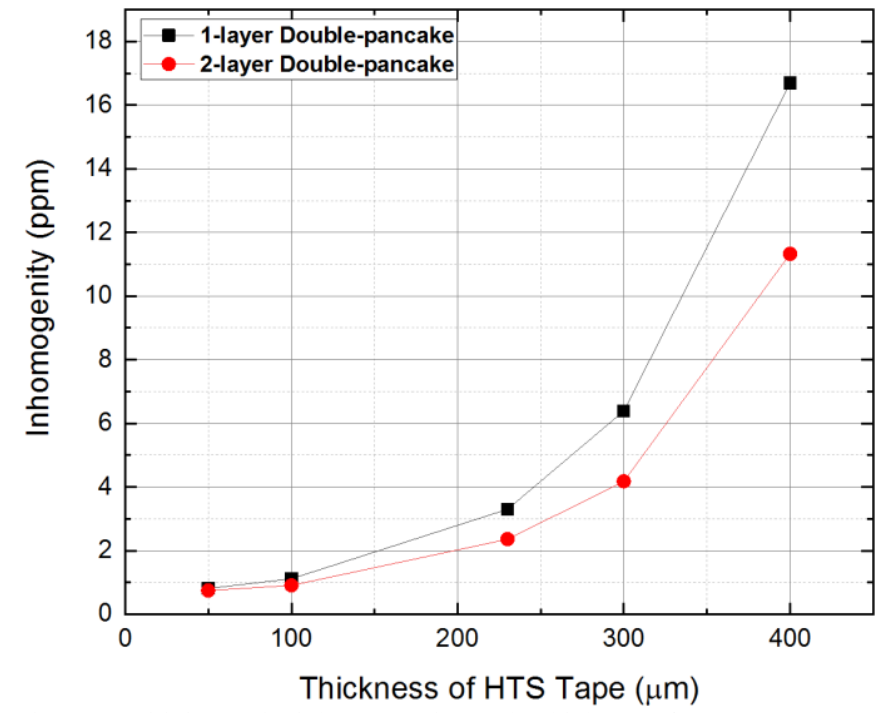

Fig. 9. Sensitivity study: inhomogeneity vs the thickness of HTS tape.

capacity, but results implies the thickness of HTS tape is a really important parameter for the uniformity optimization of magnetic field, and the tape thickness more than $400 \mu \mathrm{m}$ seems very difficult to achieve inhomogeneity less than 10 ppm.

\section{CONCLUSION}

The design of a HTS magnet was presented in order to be equipped into an ultra-compact MRI system. The design was based on the FEM package COMSOL Multiphysics with the LiveLink for MATLAB. The relatively thick HTS tape was used as the unit single element, and that brought more challenges on optimizations. Genetic Algorithm method was used to improve optimization performance, where the uniformity was able to reach $2.36 \mathrm{ppm}$ in a $10 \times 10 \times 10 \mathrm{~cm}$ DSV. Two cases of the end double-pancake were compared. The sensitivity studies were carried out on the relationship between the homogeneity and magnet length, as well as the thickness of HTS tape. It reveals that thickness of HTS tape is crucial for the uniformity optimization of magnetic field, and the thicker HTS tapes are not suitable for the design of highly uniform HTS magnet.

In the first stage, the strength and uniformity of magnetic field are the first priorities to judge the feasibility of this HTS magnet with a specific HTS tape. Other important factors such as the transient loss [23-27], anisotropic field dependence [2830], quench protection [31-33], and force analysis will be incorporated into the future design.

\section{ACKNOWLEDGMENTS}

The original platform of the Genetic Algorithm optimization based on the FEM package COMSOL Multiphysics with the LiveLink for MATLAB was developed at the School of Electronic Information and Electrical Engineering, Shanghai Jiao Tong University, China. After that, the optimization modeling was transformed and improved, and the design was accomplished at the Electrical Engineering Division, Department of Engineering, University of Cambridge. Authors would like to thank all of the members in our team for their generous supports. 


\section{REFERENCES}

[1] S. Engel, X. Zhang, and B. Wandell, "Colour tuning in human visual cortex measured with functional magnetic resonance imaging," Nature, vol. 388, no. 6637, pp. 68, 1997.

[2] Z.-P. Liang, and P. C. Lauterbur, Principles of magnetic resonance imaging: a signal processing perspective: SPIE Optical Engineering Press, 2000.

[3] S. Ogawa, T.-M. Lee, A. R. Kay, and D. W. Tank, "Brain magnetic resonance imaging with contrast dependent on blood oxygenation," Proceedings of the National Academy of Sciences, vol. 87, no. 24, pp. 9868-9872, 1990.

[4] P. Metherall, D. Barber, R. Smallwood, and B. Brown, "Threedimensional electrical impedance tomography," Nature, vol. 380, no. 6574, pp. 509, 1996.

[5] D. Holder, Electrical impedance tomography: methods, history and applications: CRC Press, 2004.

[6] B. Shen, L. Fu, J. Geng, X. Zhang, H. Zhang, Q. Dong, C. Li, J. Li, and T. A. Coombs, "Design and simulation of superconducting Lorentz Force Electrical Impedance Tomography (LFEIT)," Phys. C, Supercond., vol. 524, pp. 5-12, 2016.

[7] B. Shen, J. Geng, C. Li, X. Zhang, L. Fu, H. Zhang, J. Ma, and T. Coombs, "Optimization study on the magnetic field of superconducting Halbach Array magnet," Phys. C, Supercond., vol. 538, pp. 46-51, 2017.

[8] B. Shen, L. Fu, J. Geng, H. Zhang, X. Zhang, Z. Zhong, Z. Huang, and T. A. Coombs, "Design of a Superconducting Magnet for Lorentz Force Electrical Impedance Tomography," IEEE Trans. Appl. Supercond., vol. 26, no. 3, 2016.

[9] B. A. Glowacki, W. J. Nuttall, and R. H. Clarke, "Beyond the helium conundrum," IEEE Trans. Appl. Supercond., vol. 23, no. 3, pp. 05001130500113, 2013.

[10] F. Byrne, J. Davies, and C. Raynor, "An HTS magnet for whole-body MRI," in Proc. EUCAS, 1999.

[11] T. Nakashima, S. Kobayashi, T. Kagiyama, K. Yamazaki, M. Kikuchi, S. Yamade, K. Hayashi, K. Sato, J. Shimoyama, and H. Kitaguchi, "Recent R\&D progress on DI-BSCCO wires with high critical current properties," Phys. C, Supercond., vol. 471, no. 21-22, pp. 1086-1089, 2011.

[12] K.-i. Sato, S.-i. Kobayashi, and T. Nakashima, "Present status and future perspective of bismuth-based high-temperature superconducting wires realizing application systems," Japanese Journal of Applied Physics, vol. 51, no. 1R, pp. 010006, 2011.

[13] Y. Terao, O. Ozaki, C. Ichihara, S. Kawashima, T. Hase, H. Kitaguchi, S.-i. Kobayashi, K.-i. Sato, I. Nakajima, and N. Oonishi, "Newly designed 3 T MRI magnet wound with Bi-2223 tape conductors," IEEE Trans. Appl. Supercond., vol. 23, no. 3, pp. 4400904-4400904, 2013.

[14] B. Parkinson, "Design considerations and experimental results for MRI systems using HTS magnets," Supercond. Sci. Technol., vol. 30, no. 1, pp. 014009, 2016.

[15] B. J. Parkinson, R. Slade, M. J. Mallett, and V. Chamritski, "Development of a cryogen free 1.5 T YBCO HTS magnet for MRI," IEEE Trans. Appl. Supercond., vol. 23, no. 3, pp. 4400405-4400405, 2012.

[16] H. Maeda, and Y. Yanagisawa, "Recent developments in hightemperature superconducting magnet technology," IEEE Trans. Appl. Supercond., vol. 24, no. 3, pp. 1-12, 2013.

[17] M. Parizh, Y. Lvovsky, and M. Sumption, "Conductors for commercial MRI magnets beyond NbTi: requirements and challenges," Supercond. Sci. Technol., vol. 30, no. 1, pp. 014007, 2016.

[18] L. Davis, "Handbook of genetic algorithms," 1991.

[19] J. McCall, "Genetic algorithms for modelling and optimisation," Journal of Computational and Applied Mathematics, vol. 184, no. 1, pp. 205222, 2005.

[20] H. Zhang, J. Geng, B. Shen, X. Zhang, M. Baghdadi, and T. Coombs, "Uniform Magnetic Field Mapping With HTS Coils: Conceptual Design and Optimization," IEEE Trans. Appl. Supercond., vol. 26, no. 3, pp. 1$5,2016$.

[21] N. R. Shaw, and R. E. Ansorge, "Genetic algorithms for MRI magnet design," IEEE Trans. Appl. Supercond., vol. 12, no. 1, pp. 733-736, 2002.

[22] Q. Wang, G. Xu, Y. Dai, B. Zhao, L. Yan, and K. Kim, "Design of open high magnetic field MRI superconducting magnet with continuous current and genetic algorithm method," IEEE Trans. Appl. Supercond., vol. 19, no. 3, pp. 2289-2292, 2009.
[23] B. Shen, C. Li, J. Geng, X. Zhang, J. Gawith, J. Ma, Y. Liu, F. Grilli, and T. Coombs, "Power dissipation in HTS coated conductor coils under the simultaneous action of AC and DC currents and fields," Supercond. Sci. Technol., vol. 31, no. 7, 2018.

[24] J. Xia, H. Bai, J. Lu, A. V. Gavrilin, Y. Zhou, and H. W. Weijers, "Electromagnetic modeling of REBCO high field coils by the Hformulation," Supercond. Sci. Technol., vol. 28, no. 12, pp. 125004, 2015.

[25] B. Shen, J. Li, J. Geng, L. Fu, X. Zhang, H. Zhang, C. Li, F. Grilli, and T. A. Coombs, "Investigation of AC losses in horizontally parallel HTS tapes," Supercond. Sci. Technol., vol. 30, no. 7, 2017.

[26] B. Shen, J. Li, J. Geng, L. Fu, X. Zhang, C. Li, H. Zhang, Q. Dong, J. $\mathrm{Ma}$, and T. A. Coombs, "Investigation and comparison of AC losses on Stabilizer-free and Copper Stabilizer HTS tapes," Phys. C, Supercond., vol. 541, 2017.

[27] B. Shen, F. Grilli, and T. Coombs, "Review of the AC Loss Computation for HTS using H formulation," Supercond. Sci. Technol., 2020.

[28] N. Strickland, and S. Wimbush, "The magnetic-field dependence of critical current: What we really need to know," IEEE Trans. Appl. Supercond., vol. 27, no. 4, pp. 1-5, 2016.

[29] D. Turrioni, E. Barzi, M. J. Lamm, R. Yamada, A. Zlobin, and A. Kikuchi, "Study of HTS wires at high magnetic fields," IEEE Trans. Appl. Supercond., vol. 19, no. 3, pp. 3057-3060, 2009.

[30] P. Leys, M. Klaeser, F. Schleissinger, and T. Schneider, "Analysis of the anisotropic critical current behaviour of HTS coated conductors." p. 022013.

[31] H. Weijers, U. Trociewitz, W. Markiewicz, J. Jiang, D. Myers, E. Hellstrom, A. Xu, J. Jaroszynski, P. Noyes, and Y. Viouchkov, "High field magnets with HTS conductors," IEEE Trans. Appl. Supercond., vol. 20, no. 3, pp. 576-582, 2010.

[32] E. A. Young, C. M. Friend, and Y. Yang, "Quench characteristics of a stabilizer-free 2G HTS conductor," IEEE Trans. Appl. Supercond., vol. 19, no. 3, pp. 2500-2503, 2009.

[33] H. Weijers, W. Markiewicz, A. Voran, S. Gundlach, W. Sheppard, B. Jarvis, Z. Johnson, P. Noyes, J. Lu, and H. Kandel, "Progress in the development of a superconducting $32 \mathrm{~T}$ magnet with REBCO high field coils," IEEE Trans. Appl. Supercond., vol. 24, no. 3, pp. 1-5, 2013. 